\title{
Intraglomerular Lesions of Renal Angiomyolipoma in Tuberous Sclerosis Complex: A Case Report
}

\author{
Florencia Von Stecher, MD ${ }^{1}$, Yanina Monges, MD ${ }^{1}$, Graciela De Rosa, MD ${ }^{1}$ \\ ${ }^{1}$ Department of Pathology, Hospital De Clínicas “José De San Martín”, Buenos Aires, Argentina. \\ fvonstecher@gmail.com \\ *Corresponding Author: Florencia Von Stecher, Gorriti 3633, $1^{\text {st }}$ floor, Apartment C, 1172, Buenos Aires, \\ Argentina.
}

\begin{abstract}
The association between tuberous sclerosis complex (TSC) and renal angiomyolipomas (RAML) is widely recognized, but the evidence of intraglomerular microlesions of angiomyolipoma in TSC is extremely rare. We report a 36-year-old woman with TSC, presenting as gross hematuria and hypovolemic shock. The patient was diagnosed with bilateral RAML and spontaneous rupture of right RAML. She underwent right nephrectomy. Microscopic examination revealed multiple bilateral RAML composed of dysmorphic blood vessels, smooth muscle, epithelioid cells and adipose tissue, as well as intraglomerular microlesions of these components in continuity with the glomerular basement membrane but without adhesion to the Bowman's capsule. In this report we describe the histopathologic features and immunohistochemical markers of TSC-associated RAML with multiple parenquimatous lesions and isolated micro foci in the glomerular tuft. We also review recent discoveries related to possible pathogenesis of renal and intraglomerular lesions.
\end{abstract}

Keywords: angiomyolipoma, kidney, intraglomerular, Wunderlich's syndrome

\section{INTRODUCTION}

Tuberous sclerosis complex (TSC) is an autosomal dominant disease with a mean incidence estimated in $1 / 6000$ live births ${ }^{1}$. Approximately $70-85 \%$ of patients with TSC develop renal angiomyolipomas (RAML). The most common complication of RAML is Wunderlich's syndrome, an urological emergency due to spontaneous, non-traumatic retroperitoneal hemorrhage, which occurs in $10 \%$ of the cases and can be a life-threatening condition ${ }^{2}$. Pathologically, RAML are characterized by variable admixture of adipocytes, smooth muscle cells and abnormal vasculature ${ }^{3}$. The presence of intraglomerular microlesions is extremely rare and there are a few number of reports in the literature ${ }^{4}$.

\section{CASE REPORT}

A 36-year-old woman with medical history of TSC and intellectual disability was admitted to our hospital with sudden-onset right-sided abdominal pain, gross hematuria and hypotension. Abdominal computed tomography (CT) revealed multiple and bilateral renal masses with heterogeneous fat density, which were consistent with RAML. The right kidney showed a large heterogeneous tumor measuring $12.7 \mathrm{x} 10.5 \mathrm{x}$ $9.5 \mathrm{~cm}$, associated with haematoma, suggestive of ruptured RAML.

A diagnosis of bilateral, multifocal RAML with Wunderlich's syndrome was made. After the patient was hemodynamically stable, right nephrectomy was performed. No complications were observed and the patient was discharged on the fourth postoperative day in a good condition.

Macroscopic examination revealed an enlarged kidney, measuring 14x 8x $6 \mathrm{~cm}$, with multiple nodules range from $0.5-2 \mathrm{~cm}$ in diameter and a solid cystic tumor measuring 9.8x 7.5x $6 \mathrm{~cm}$ associated with a large perirenal hematoma.

Histologically, the tumors were composed of thickwalled, hyalinised vessels, many of them without 
Intraglomerular Lesions of Renal Angiomyolipoma in Tuberous Sclerosis Complex: A Case Report

internal elastic lamina, bundles of smooth muscle, mature adipose tissue and epithelioid cells of varying proportions. Necrosis and hemorrhage were also observed. Numerous microscopic tumor foci were found throughout the parenchyma, as well as isolated intraglomerular segmental nodules consisting of epithelioid, smooth muscle and adipose cells without attachment to the Bowman's capsule (Fig. 1).

Neoplastic cells expressed melanocytic markers (HMB-45 and Melan-A), desmin, smooth muscle actin, vimentin and were negative for pan-cytokeratin AE1AE3.

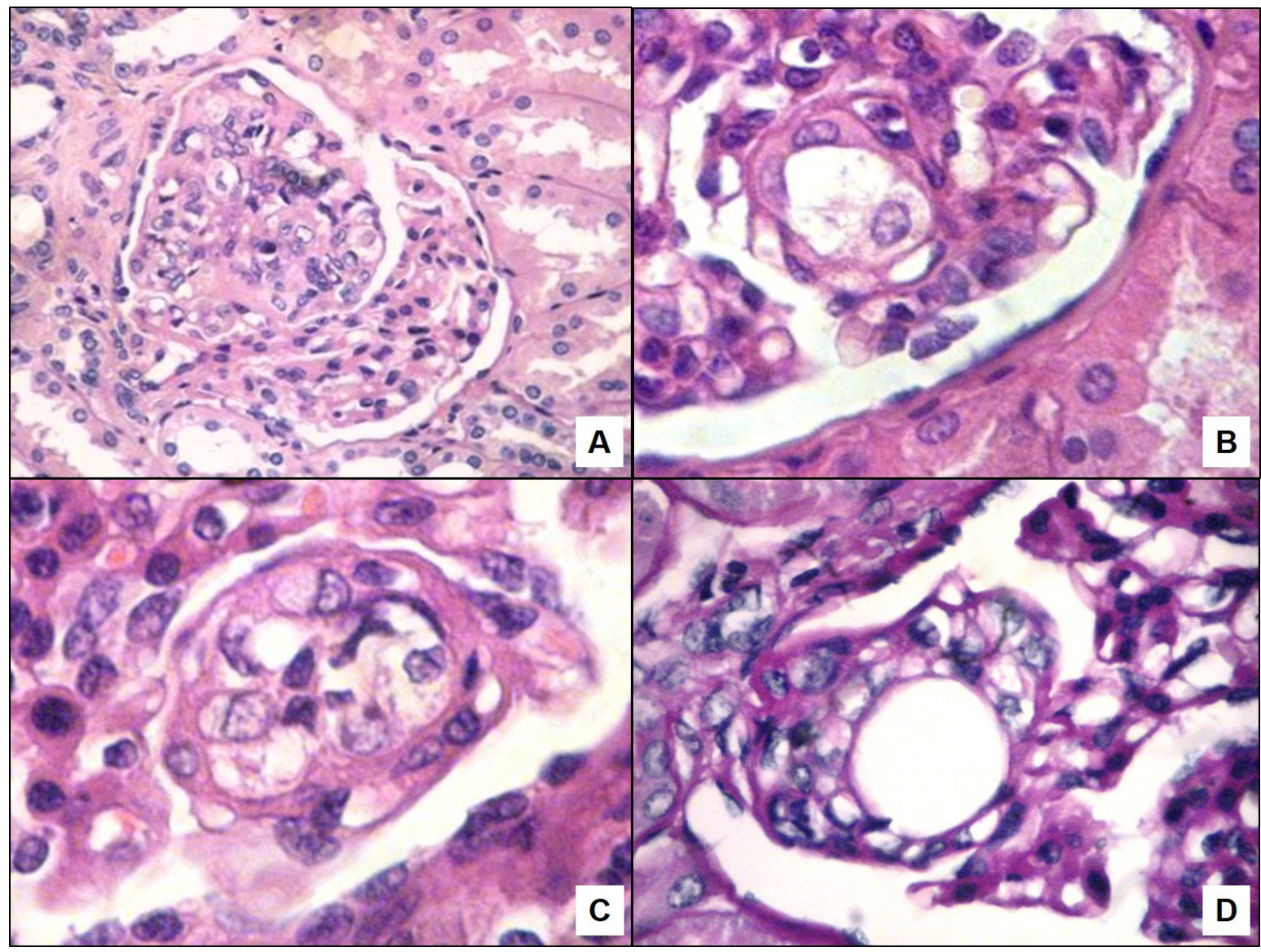

Figure 1. Intraglomerular microlesions of angiomyolipoma consisting of adipose and epithelial cells. A-C: hematoxylin-eosin; original magnification, 200x (A) and 1000x (B-C). D: periodic acid-Schiff; original magnification, $1000 x$

\section{Discussion}

TSC is an autosomal dominant disease characterized by hamartomatous tumors of the skin, brain, kidney, heart and lung ${ }^{3}$. TSC is caused by mutations in two genes, TSC1 at position 9q34 and TSC2 at 16p13.35, which encode proteins that act as tumor suppressors. These proteins are called hamartin and tuberin, respectively. Kidneys are frequently involved in TSC and renal complications are the most common causes of morbidity and mortality in these patients. Renal disease includes angiomyolipomas, epithelial cysts and renal cell carcinoma ${ }^{6}$. Multiple and bilateral RAML develop in up to $80 \%$ of patients with $\mathrm{TSC}^{7}$. Although these tumors are usually asymptomatic, lesions larger than $3 \mathrm{~cm}$ in diameter are prone to bleeding. The risk of bleeding is proportional to the size of the lesion and up to $10 \%$ of TSC patients may experiment spontaneous massive and potentially fatal retroperitoneal hemorrhage or Wunderlich's syndrome ${ }^{8}$.

This report describes a case of bilateral RAML with multiple nodular lesions either inside or outside the glomeruli. All glomerular lesions were in continuity with the capillary wall. The lack of attachment to the Bowman's capsule suggests that the origin of these microlesions occurs inside the glomerulus. 
Intraglomerular Lesions of Renal Angiomyolipoma in Tuberous Sclerosis Complex: A Case Report

The intraglomerular microlesions in RAML were first reported in 1930 by Feriz et $\mathrm{al}^{9}$. They described "epithelial and fatty inclusions" in the glomerular tufts. In 1973, Shinohara et $\mathrm{a}^{10}$ reported similar lesions, but the first detailed description was given by Nagashima et al in $1988^{11}$.

The RAML are histologically classified as typical (triphasic) or atypical (monophasic or epithelioid). Most RAML contain variable admixtures of tumor cells that are histologically and molecularly similar to vascular, smooth muscle and fat lineages ${ }^{12}$. Renal AML cells express molecular markers of melanocyte lineage such as HMB-45 and melan-A and muscular markers such as smooth muscle actin and less frequently desmin $^{13}$. It has been proposed that they might originate from undifferentiate cells of the neural crest or of an unidentified AML neoplastic stem cell ${ }^{14}$.

RAML have been considered to be an hamartoma for a long time, but there is strong evidence supporting that they are true neoplasms with a clonal origin ${ }^{15-}$ ${ }^{17}$. Martignoni et $\mathrm{al}^{15}$ suggested that there is a group of lesions that originate from a progenitor cell called perivascular epithelioid cell (PEC) which is characterized by an epithelioid appearance, perivascular distribution and intracytoplasmic HMB45 positivity. They described intraglomerular lesions with features of angiomyolipoma in patients with and without tuberous sclerosis and in the TSC2/PKD1 contiguous gene syndrome, a disease with a deletion disrupting both TSC2 and PKD1 (autosomal dominant polycystic disease gene).

Recently, Gonçalves et $\mathrm{al}^{17}$ described the first mouse model of RAML and provided evidence that these mesenchymal tumors originate from renal proximal tubule epithelial cells.

\section{CONCLUSION}

The intraglomerular microlesions of angiomyolipoma are extremely infrequent. Human RAML express melanocyte and smooth-muscle markers, as we found in our case. However, the accurate cell origin of AML or AML stem cell remain unknown.

\section{REFERENCES}

[1] Crino PB, Nathanson KL, Hensk EP. The tuberous sclerosis complex. $N$ Engl J Med. 2006;355(13):1345-1356.
[2] Sooriakumaran P, Gibbs P, Coughlin G, et al. Angiomyolipomata: Challenges, solutions, and future prospects based on over 100 cases treated. BJU Int. 2010;105(1):101-106.

[3] Lam HC, Siroky BJ, Henske EP. Renal disease in tuberous sclerosis complex: pathogenesis and therapy. Nat Rev Nephrol. 2018;14(11):704-716.

[4] Kiliçaslan I, Güllüoglu MG, Dogan O, Uysal $\mathrm{V}$. Intraglomerular microlesions in renal angiomyolipoma.HumPathol.2000;31(10):13251328.

[5] Borkowska J, Schwartz RA, Kotulska K, Jozwiak S. Tuberous sclerosis complex: tumors and tumorigenesis.Int J Dermatol. 2011;50(1):13-20.

[6] Bissler JJ, Christopher Kingswood J. Renal manifestation of tuberous sclerosis complex. Am J Med Genet C Semin Med genet. 2018;178(3):338347.

[7] Gipson TT. Consequences of delay in screening, monitoring, and treatment of angiomyolipoma and tuberous sclerosis: A case report. Clin Nephrol. 2018;90(1):71-75.

[8] Moratalla MB. Wunderlich's syndrome due to spontaneous rupture of large bilateral angiomyolipomas. EmergMed J. 2009;26(1):72.

[9] Feriz H: Ein Beitragzur Histopathologie der tuberösen Sklerose. Virchows Archiv Für Pathologische Anatomie Und Physiologie Und Für Klinische Medizin. 1930;278(3):690-769.

[10] Shinohara S, Kosugi Y, Fujitani K, et al. Clinical aspects of tuberous sclerosis. Early symptoms and electroencephalogram of patients and their relatives (in Japanese). BrainNerve. 1973;25:1317-1334.

[11] Nagashima Y, Ohaki Y, Tanaka Y, Misugi K, Horiuchi M. A case of renal angiolipomas associated with multiple and various hamartomatous microlesions. Virchows Archiv A Pathol Anat. 1988;413(2):177-182.

[12] Vos N, Oyen R. Renal Angiomyolipoma: The good, the bad, and the ugly. J Belg Soc Radiol. 2018;102(1):41.

[13] Esheba Gel S, Esheba Nel S. Angiomyolipoma of the kidney: clinic opathological and 
Intraglomerular Lesions of Renal Angiomyolipoma in Tuberous Sclerosis Complex: A Case Report

immunohistochemical study. J Egypt Natl Canc Inst. 2013;25(3):125-134

[14] Fernandez-Flores, A. Evidence on the neural crest origin of PEComas. Rom J Morphol Embryol. 2011;52(1):7-13.

[15] Martignoni G, Pea M, Reghellin D, Zamboni G, Bonetti F. Perivascular epithelioid cell tumor (PEComa) in the genitourinary tract. Adv Anat Pathol. 2007;14(1):36-41.
[16] Cheng L, Gu J, Eble JN, et al. Molecular genetic evidence for different clonal origin of components of human renal angiomyolipomas. Am J Surg Pathol. 2001;25(10):1231-1236.

[17] Gonçalves AF, Adlesic M, Brandt S, et al. Evidence of renal angiomyolipoma neoplastic stem cells arising from renal epithelial cells. Nat Commun. 2017;8(1):1466.

Citation: Florencia Von Stecher, MD, Yanina Monges, MD, Graciela De Rosa, MD. Intraglomerular Lesions of Renal Angiomyolipoma in Tuberous Sclerosis Complex: A Case Report. Archives of Urology. 2019; 2(2): 32-35.

Copyright: (C) 2019 Florencia Von Stecher, MD, Yanina Monges, MD, Graciela De Rosa, MD. This is an open access article distributed under the Creative Commons Attribution License, which permits unrestricted use, distribution, and reproduction in any medium, provided the original work is properly cited. 